\title{
Farmacocinética das estatinas
}

\section{Pharmacokinetics of statins}

Francisco Antonio Helfenstein Fonseca.

Setor de Lípides, Aterosclerose e Biologia Vascular, Disciplina de Cardiologia UNIFESP - EPM.

As estatinas são agentes hipolipemiantes que exercem os seus efeitos através da inibição da HMG-CoA redutase, enzima fundamental na síntese do colesterol, levando a uma redução do colesterol tecidual e um conseqüente aumento na expressão dos receptores de LDL. Existem consideráveis diferenças entre as estatinas, no que tange às propriedades farmacocinéticas, bem como ao coeficiente de hidrofilicidade, via hepática de metabolização (especialmente, do citocromo P450 e isoenzimas), meia-vida plasmática e eficácia na redução lipídica. As estatinas também podem diferir na capacidade de interação com outras drogas que utilizam a mesma via de metabolização. Recentemente, muitos efeitos pleiotrópicos têm sido relatados com estas drogas, bem como propriedades antiinflamatórias, melhora na função endotelial e benefícios na hemostasia.

\section{Palavras-chave}

Estatinas, farmacocinéticas, interação com drogas
Statins are lipid lowering agents that promote their effects on plasma lipids through the inhibition of HMGCoA reductase, a crucial enzyme in the cascade of cholesterol synthesis, leading to reduction of tissue cholesterol pool and consequently, to an upregulation of the $L D L$ receptor expression. There are considerable differences among statins regarding some pharmacokinetic properties, such as the coefficient of hydrophilicity, via liver metabolism (especially regarding P450 cytochrome and isoenzymes), plasma half-life and efficacy of serum lipid changes. They may also differ regarding interactions with other drugs that share the same pathway of metabolism. Recently, many pleiotropic effects have been reported with these drugs, such as anti-inflammatory properties, improvement in endothelial function and benefits on hemostasis.

\section{KEY WORDS}

Statins, pharmacokinetics, drug interactions
Os inibidores da HMG-CoA redutase (vastatinas ou estatinas) constituem uma notável classe de medicamentos redutores de colesterol e têm sido associados com uma expressiva diminuição da morbidade e mortalidade cardiovascular para pacientes em prevenção primária ou secundária da doença coronariana ${ }^{1}$ (Figura 1 ).

\section{Redução de eventos coronarianos com estatinas}

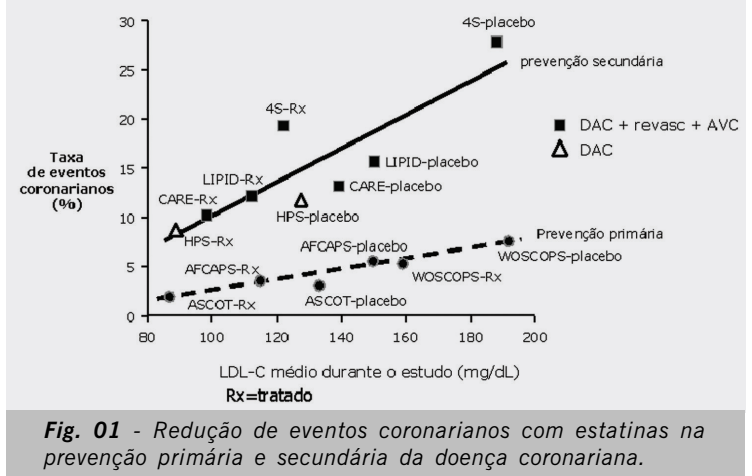

Entretanto, a questão que permaneceu após todos estes estudos é a de que apesar dos benefícios, um considerável resíduo de morbidade e mortalidade ainda foi observado, sugerindo que uma redução adicional do colesterol pudesse estar associada com vantagens adicionais. Após a publi- cação em 2001 das diretrizes propostas pelo National Cholesterol Education Program Adult Treatment Panel (NCEP-ATP III) sucessivos estudos vêm confirmando esta hipótese, e de fato, uma redução adicional nas metas de LDL-C acabou sendo sugerida em $2004^{2}$.

A figura 2 mostra a relação log linear entre o risco de doença arterial coronariana e o valor do LDL-C².

\section{Relação log linear entre LDL-c e RR da DAC}

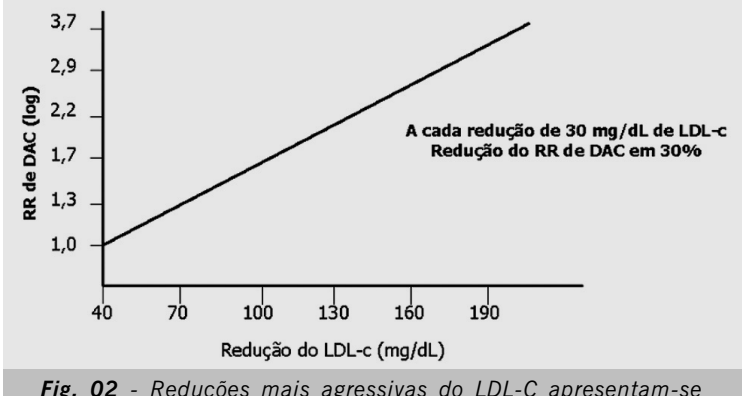

Fig. 02 - Reduções mais agressivas do LDL-C apresentam-se correlacionadas com a redução de desfechos coronarianos.

A necessidade de metas mais agressivas de redução do LDL-C determina a utilização de estatinas de primeira geração em doses mais altas ou o uso de fármacos mais recentes e mais efetivos. Neste contexto, torna-se essencial o conhecimento das propriedades farmacocinéticas e farma- 
codinâmicas desta classe de medicamentos.

O mecanismo de ação das estatinas para obtenção da redução do colesterol se deve a inibição da enzima HMGCoA redutase, por meio de uma afinidade destes fármacos com o sítio ativo da enzima (Figura 3). Esta inibição é reversível e competitiva com o substrato $\mathrm{HMG}-\mathrm{CoA}^{3}$.

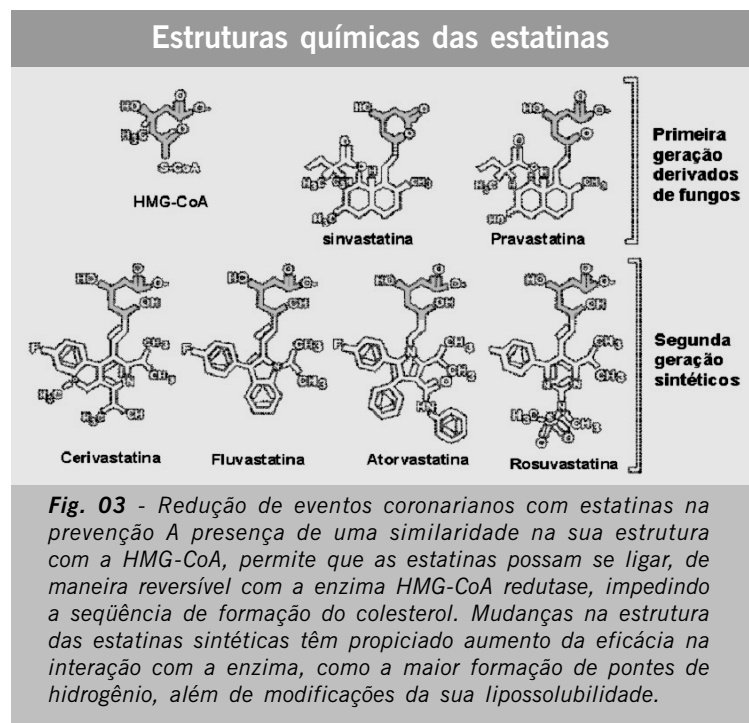

Estudos em humanos ${ }^{3}$ mostraram que a magnitude desta inibição difere substancialmente entre as estatinas, como mostrado na figura 4 .

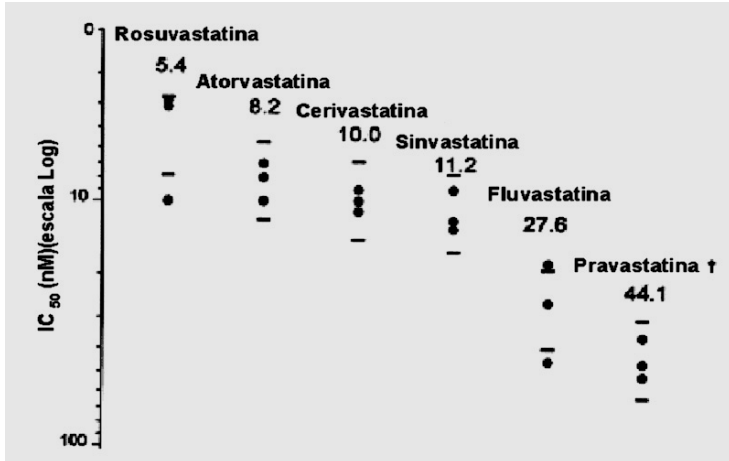

Fig. 04 - Inibição da atividade da HMG-CoA redutase mostrando as diferenças entre as estatinas. Os valores representam experimentos em humanos com três determinações e as barras representam um intervalo de confiança de $95 \%$.

Algumas estatinas possuem uma grande seletividade de atuação, principalmente como decorrência de características como a lipossolubilidade. Neste aspecto, verifica-se que a pravastatina e a rosuvastatina são fármacos que podem ser considerados relativamente hidrossolúveis, quando comparados com as demais estatinas. (Figura 5)

As estatinas atuam primariamente no fígado, onde um sistema especial de transporte permite sua incorporação ao tecido hepático para biotransformação, com ampla variação tanto no seu sítio metabólico, como formação de metabólitos ativos até sua eliminação pela bile. Alguns polimorfismos genéticos e a interação com alguns fármacos podem determinar dificuldade na sua captação, metabolização e eliminação.

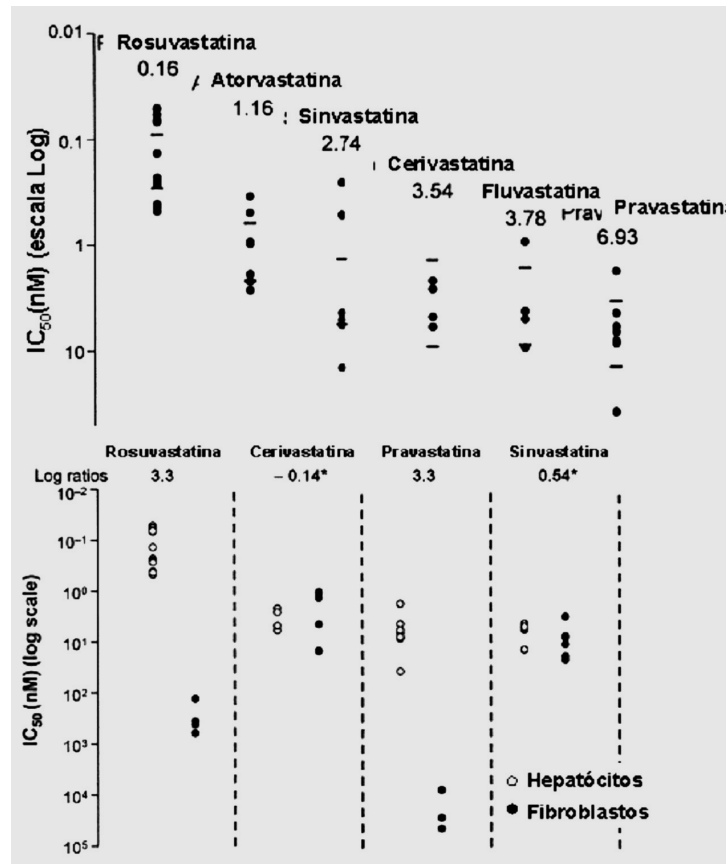

Fig. 05 - Na figura acima, valores obtidos para a inibição da síntese de colesterol em hepatócitos de ratos. Na figura abaixo, a comparação entre os valores de $I C_{50}$ obtidos em hepatócitos e fibroblastos, mostrando importantes diferenças na seletividade tecidual entre as estatinas.

O transporte para o tecido hepático parece influenciado pelos OATPs (Organic Anion Transporting Polypeptides), uma família de transportadores expressa em vários órgãos (Figura 6). No fígado o OATP-C constitui um membro específico desta superfamília e sua expressão foi confirmada por imunohistoquímica na membrana basolateral dos hepatócitos ${ }^{4}$. O OATP-C transporta várias substâncias, como ácidos biliares, conjugados glucuronidados, hormônios tireoidianos, peptídios, metotrexate e estatinas, incluindo a pravastatina, rosuvastatina e cerivastatina (comprovado) e atorvastatina (possível). Interações farmacológicas que reduzam a disponibilidade do OATP-C ou polimorfismos genéticos que diminuam sua expressão podem determinar redução da efetividade destas estatinas.

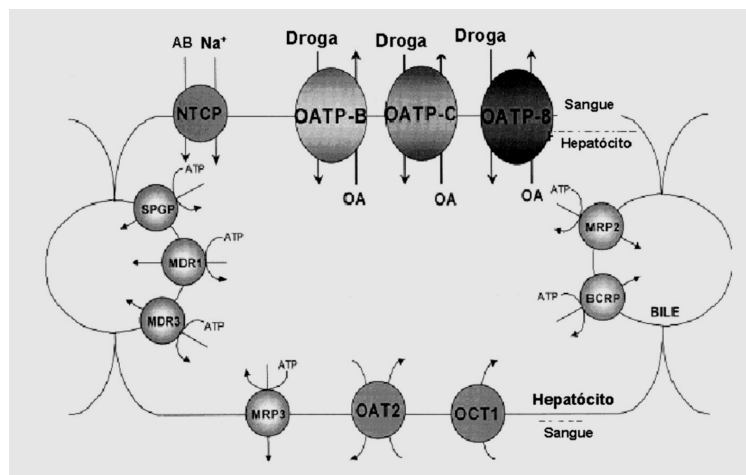

Fig. 06 - Esquema de captação e efluxo de transportadores expressos em hepatócitos humanos, de importância para o metabolismo hepático de vários fármacos. $A B=$ ácidos biliares; OATP = organic anion transporting polypeptide; $M D R=$ multiple drug resistance; $A T P=$ adenosina trifosfato; $M R P=$ multidrug resistance protein; $O C T 1=$ organic cátion transporter $1 ; B C R P=$ breast câncer resistance protein; SPGP = sister of P-glycoprotein. 
Após sua biotransformação, a eliminação das estatinas também pode ser influenciada por outro sistema transportador (glicoproteína-P), responsável pelo efluxo para a árvore biliar (gene MDR1- Multiple Drug Resistance), e novamente algumas interações entre fármacos ou polimorfismos também podem determinar acúmulo da vastatina no tecido hepático ou sua mais rápida eliminação. Assim, alguns inibidores de protease, digoxina, ciclosporina, entre outros, podem interferir na excreção hepática das estatinas ${ }^{5}$.

Um dos aspectos mais descritos com as estatinas é o seu potencial de interações farmacológicas com drogas que compartilham o mesmo sítio de metabolização microssomal hepático. Assim, nos EUA foi descrito um número muito baixo de complicações graves, como a rabdomiólise, uma síndrome caracterizada por necrose muscular, elevação de creatinofosfoquinase (CPK) acima de 10 vezes o limite superior da normalidade (LSN), acompanhada de dores musculares, mioglobinúria e determinando, em geral, risco de vida devido à insuficiência renal. De fato, a incidência de rabdomiólise fatal nos EUA foi descrita como sendo de 0,15 casos por milhão de prescrições de estatinas ${ }^{6}$. Quando os sintomas musculares não se acompanham de elevação significativa das enzimas musculares, caracterizamos a mialgia, que parece incidir em 6 a 14\% dos eventos adversos associados com a prescrição das estatinas ${ }^{7}$. Finalmente, miosite é o termo reservado para caracterizar a presença de sintomas musculares com elevação acima de 10 vezes o LSN.

Os mecanismos da miopatia serão abordados em outro tópico deste suplemento, mas parecem envolver alterações de membranas celulares e inibição de formação de substâncias importantes para o metabolismo celular e mitocondrial como a ubiquinona ${ }^{8,9}$.

A miopatia associada com as estatinas são dosedependentes (Figura 7) e sua incidência aumenta até cinco vezes $(\sim 0,3 \text { a } \sim 1,5 \%)^{10}$ quando certas estatinas (lovastatina, simvastatina, atorvastatina) são coadministrada com certos medicamentos como fibratos ${ }^{11}$ (especialmente gemfibrozil), bloqueadores de canais de cálcio ${ }^{12}$, imunossupressores (ciclosporina) ${ }^{13}$, agentes antifúngicos ${ }^{14}$ (itraconazol, cetoconazol, fluconazol), drogas antiretrovirais como os inibidores de protease ${ }^{15}$ e combinações destas e outras drogas, incluindo suco de grapefruit ${ }^{16}$.

Algumas das características farmacocinéticas ${ }^{17}$ destes fármacos estão presentes nas Tabelas I e II.

\begin{tabular}{|c|c|c|c|c|c|}
\hline Fármaco & $\begin{array}{l}T \max \\
\text { (h) }\end{array}$ & $\begin{array}{c}\mathrm{C} \max \\
(\mathrm{mg} / \mathrm{ml})\end{array}$ & $\begin{array}{c}\text { Substrato de } \\
\text { transportador } \\
\text { protéico }\end{array}$ & $T^{1} 1 / 2(h)$ & $\begin{array}{l}\text { Biodisponi- } \\
\text { bilidade (\%) }\end{array}$ \\
\hline Rosuvastatina & 3 & 37 & Sim & 20,8 & 20 \\
\hline Atorvastatina & $2-3$ & $27-66$ & Sim & $15-30$ & 12 \\
\hline Lovastatina & $2-4$ & $10-20$ & Sim & 2,9 & 5 \\
\hline Simvastatina & $1,3-2,4$ & $10-34$ & Sim & $2-3$ & 5 \\
\hline Fluvastatina XL & 4 & 55 & Não & 4,7 & 6 \\
\hline Pravastatina & $0,9-1,6$ & $45-55$ & Sim/Não & $1,3-2,8$ & 18 \\
\hline
\end{tabular}

\section{Tabela II. Farmacocinética das estatinas}

\begin{tabular}{|c|c|c|c|c|c|}
\hline Fármaco & $\begin{array}{l}\text { Ligação } \\
\text { protéica } \\
(\%)\end{array}$ & $\begin{array}{l}\text { Metabolismo } \\
\text { (CYP) }\end{array}$ & Metabólitos & $\begin{array}{c}\text { Excreção } \\
\text { urinária } \\
(\%)\end{array}$ & $\begin{array}{c}\text { Excreção } \\
\text { fecal } \\
(\%)\end{array}$ \\
\hline Rosuvastatina & 88 & $\begin{array}{c}\text { 2C9, 2C19 } \\
\text { (mínimo) }\end{array}$ & $\begin{array}{c}\text { Ativos } \\
\text { (mínimo) }\end{array}$ & 10 & 90 \\
\hline Atorvastatina & $80-90$ & $3 \mathrm{~A} 4$ & Ativos & 2 & 70 \\
\hline Lovastatina & $>95$ & $3 A 4$ & Ativos & 10 & 83 \\
\hline Simvastatina & $94-98$ & $3 \mathrm{~A} 4$ & Ativos & 13 & 58 \\
\hline Fluvastatina XL & $>99$ & $2 C 9$ & Inativos & 6 & 90 \\
\hline Pravastatina & $43-55$ & não & Inativos & 120 & 71 \\
\hline
\end{tabular}

Baseado em uma dose de $40 \mathrm{mg}$ oral, com a exceção da

fluvastatina XL $(80 \mathrm{mg})$.

$O$ conhecimento das vias de metabolismo dos principais fármacos utilizados no paciente com dislipidemia ou aterosclerose se torna relevante e pode auxiliar na decisão da escolha do fármaco ou dose a ser instituída. Assim, a tabela III mostra casos de rabdomiólise que foram relatados com algumas das estatinas.

$\begin{aligned} & \text { Tabela III. Relatos de rabdomiólise associados com } \\ & \text { estatinas }\end{aligned}$
$\begin{array}{ll}\text { Estatina } & \begin{array}{l}\text { Fármacos associados } \\ \text { Simvastatina }\end{array} \\ & \begin{array}{l}\text { antibióticos macrolídeos, digoxina, antifúngicos, } \\ \text { clorzoxazona, nefazodona, niacina, tacrolimus, } \\ \text { ácido fusídico }\end{array} \\ \text { Cerivastatina } & \begin{array}{l}\text { Fibratos, digoxina, warfarina, antibióticos } \\ \text { macrolídeos, ciclosporina, mibefradil }\end{array} \\ \text { Atorvastatina } & \begin{array}{l}\text { Mibefradil, fibratos, antibióticos macrolídeos, } \\ \text { warfarina, ciclosporina, digoxina, antifúngicos }\end{array} \\ \text { Pravastatina } & \begin{array}{l}\text { Fibratos, antibióticos macrolídeos, warfarin, } \\ \text { ciclosporina, digoxina, mibefradil, niacina }\end{array} \\ \text { Lovastatina } & \begin{array}{l}\text { Ciclosporina, antibióticos macrolídeos, } \\ \text { antifúngicos, fibratos, mibefradil, digoxina, } \\ \text { nefazodona, niacina, warfarina }\end{array} \\ \text { Fluvastatina } & \text { Fibratos, warfarin, digoxina, mibefradil }\end{array}$
$\begin{aligned} & \text { As interações variaram em freqüência, desde situações mais comuns, } \\ & \text { como as interaçóes com o mibefradil ou ciclosporina com várias } \\ & \text { estatinas e de fibratos com a cerivastatina, até ocorrências muito } \\ & \text { mais raras como as interações com digoxina, niacina ou warfarina. }\end{aligned}$

É interessante notar que mesmo entre fármacos com metabolização microssomial CYP450 diferenciada podem ainda ocorrer interações farmacológicas. Como exemplo, a fração livre de uma estatina pode aumentar pela adição de fibrato, pelo seu deslocamento da ligação protéica, constituindo um mecanismo para o efeito adverso descrito para a interação gemfibrozil-cerivastatina (ambos fármacos possuem alto grau de ligação protéica). Além disso, a digoxina pode interferir no transportador responsável pela excreção biliar da estatina. Assim, tendo em vista estes conceitos a Tabela IV contém alguns medicamentos de uso freqüente em pacientes com doença cardiovascular que apresentam potencial para interação farmacológica.

Com relação à interação de fibratos com estatinas tem sido relatado que o gemfibrozil aumenta a concentração de formas ativas de sinvastatina e lovastatina, mas minimamente a forma lactônica. Recentemente, foi mostrado que o gemfibrozil modula a farmacocinética das estatinas principalmente via inibição 


\begin{tabular}{|c|c|c|c|c|c|}
\hline CYP1A2 & CYP2C9 & CYP2C19 & CYP2D6 & CYP2E1 & CYP3A4 \\
\hline Acetaminofen & Alprenolol & Diazepam & Amitriptilina & Acetaminofen & Amiodarona \\
\hline Cafeína & Diclofenaco & Ibuprofen & Codeína & Etanol & Atorvastatina \\
\hline \multirow[t]{16}{*}{ Teofilina } & Fluvastatina & Mefenitoína & Debrisoquina & halotano & Claritromicina \\
\hline & Fenitoina & Metilfenobarbital & Flecainida & & Ciclosporina \\
\hline & Tolbutamida & Omeprazol & Imipramina & & Diltiazem \\
\hline & Warfarina & Fenitoína & Metoprolol & & Eritromicina \\
\hline & & & Mibefradil & & Itraconazol \\
\hline & & & Nortriptilina & & Cetoconazol \\
\hline & & & Propafenona & & Lacidipina \\
\hline & & & Timolol & & Lovastatina \\
\hline & & & & & Mibefradil \\
\hline & & & & & Nifedipina \\
\hline & & & & & Inibidores de protease \\
\hline & & & & & Quinidina \\
\hline & & & & & Sildenafil \\
\hline & & & & & Terbinafina \\
\hline & & & & & Verapamil \\
\hline & & & & & Warfarina \\
\hline
\end{tabular}

da glucuronidação e, como esperado, com pouca interferência na inibição do CYP3A4 ${ }^{18,19}$. Estes aspectos mostram como uma interação ao nível de transporte pode afetar o metabolismo de estatinas que não compartilham uma mesma via de metabolização (como a interação gemfibrozil-pravastatina). Além disso, fibratos que não alteram ou o fazem minimamente neste sistema, como o fenofibrato, apresentam menor potencial de interação com o metabolismo das estatinas ${ }^{20}$.

Além disso, expressivo aumento nas concentrações séricas da pravastatina foram descritas na presença de ciclosporina A, por redução do clearance biliar da estatina determinada pela interferência no sistema transportador através da membrana canalicular biliar ou ainda no sistema da glicoproteína P. Assim, mesmo para as estatinas de menor potencial para interações farmacológicas, como a pravastatina, ainda podem ser observadas situações de risco, onde um adequado acompanhamento e a titulação inicial de doses mais baixas das estatinas podem ser fundamentais.

Entretanto, é importante não apenas conhecer alguns dos substratos que utilizam uma via comum de metabolização, mas também a capacidade de algumas destas substâncias inibirem ou induzirem a via enzimática de metabolização. Devido ao grande número de fármacos que apresentam a via de biotransformação do CYP3A4 e 2C9 e sua importância para algumas das estatinas, inserimos na tabela $\mathrm{V}$, fármacos que podem modificar 0 metabolismo destas estatinas.

Apesar das potenciais interações entre as estatinas e alguns fármacos de uso rotineiro na prática clínica, efeitos adversos são relativamente raros tanto em nível hepático como muscular, embora sejam dose-dependentes, como mostrado nas Figuras 7 e 8.

Sumarizando, as estatinas apresentam muitas diferenças farmacocinéticas entre si. Os efeitos adversos são dose- dependentes e muitos fármacos podem modificar seu metabolismo, expondo o paciente a um maior risco. Tendo em vista a ampliação das indicações e as metas mais rigorosas torna-se importante um maior conhecimento das propriedades farmacocinéticas destes fármacos.

Do ponto de vista farmacodinâmico, as estatinas são bastante seletivas para sua atuação junto à enzima HMGCoA redutase e vários dos efeitos pleiotrópicos destes fármacos parecem depender de uma menor ativação de

\begin{tabular}{l|l|l}
\multicolumn{3}{|c}{ Tabela V. Inibidores e indutores da via } \\
do citocromo P450
\end{tabular}


Hepatopatia relacionada ao uso das estatinas

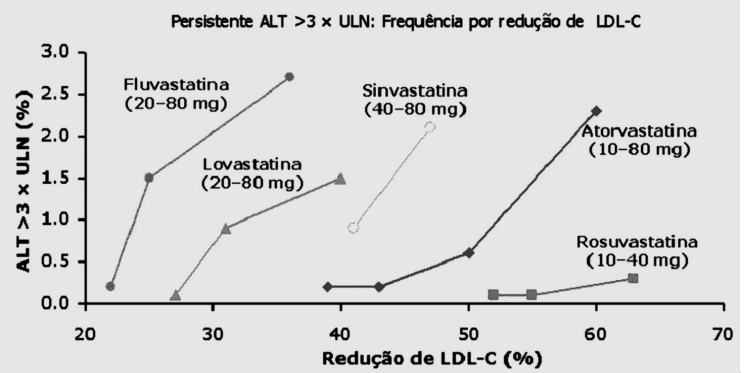

Fig. 07 - Hepatopatia relacionada com o uso de estatinas. Foram computadas as alterações que incidiram em pelo menos duas dosagens com base em estudos clínicos. Observa-se que o aumento da dose (relacionada com a maior redução do $L D L-C$ ) associa-se com o aumento da hepatotoxicidade.

\section{Miopatia associada com 0 uso das estatinas}

CK >10 x LSN: Frequência por redução de LDL-C

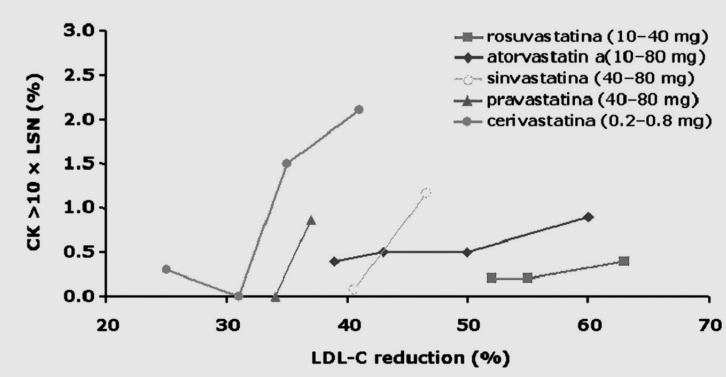

Fig. 08 - A incidência de miopatia pode ser considerada relativamente infrequente, porém relacionada ao emprego de doses mais elevadas para todas as estatinas.

algumas proteínas que interferem em vários e importantes vias de sinalização celular, relacionadas a genes que condicionam a síntese de citocinas inflamatórias, fatores de coagulação, ou relacionados a maior expressão de óxido nítrico. Assim, a redução de mevalonato determina menor ativação das proteínas Ras e Rho, promovendo efeitos anti-inflamatórios, melhor balanço

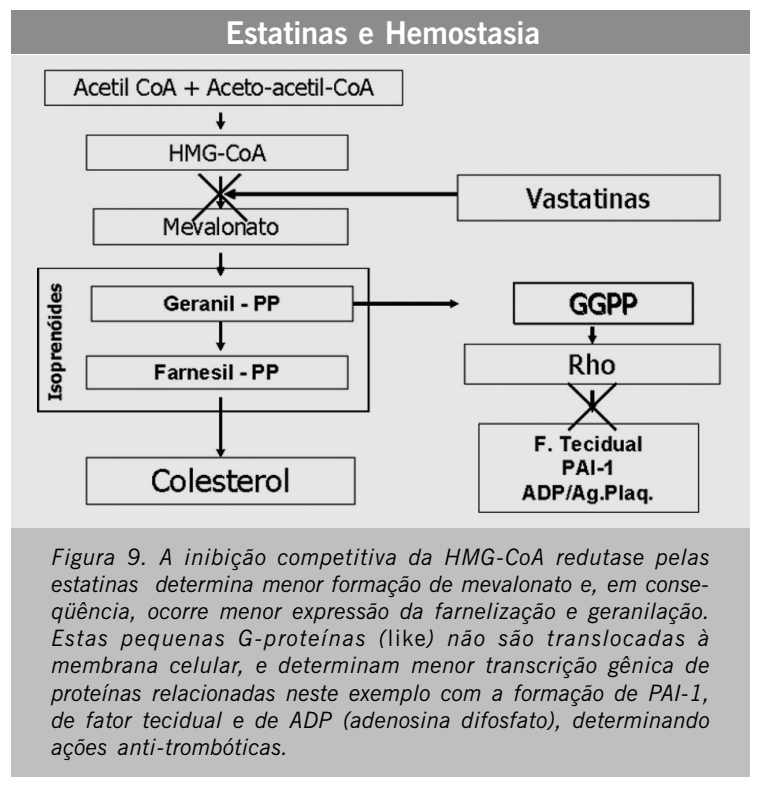

da hemostasia e recuperação da vasorreatividade dependente do endotélio ${ }^{21}$. A figura 9 exemplifica estas ações em relação à hemostasia ${ }^{22-24}$.

As ações imunomodulatórias na inflamação, a mobilização de células tronco, a diminuição da resistência à insulina, entre outras ações, tem ampliado consideravelmente as prescrições para estes fármacos, como no caso da insuficiência cardíaca ${ }^{25}$, nos transplantes ${ }^{26}$ ou na artrite reumatóide ${ }^{27}$. Além disso, cresce 0 conceito de que praticamente todos os clássicos fatores de risco podem ter seu impacto na doença aterosclerótica atenuado pelo uso das estatinas, como tem sido mostrado em estudos de hipertensão arterial ${ }^{28}$ ou diabetes ${ }^{29}$, além de situações mais recentes como indivíduos HIV em uso de antiretrovirais $^{30}$ ou em portadores de osteoporose ${ }^{21,31}$. Sendo assim, torna-se fundamental à boa prática clínica, a incorporação destes novos conceitos.

\section{REFERÊNCIAS}

1. Ballantyne CM. Clinical trial endpoints: angiograms, events, and plaque instability. Am J Cardiol. 1998;82:5M-11M

2. Grundy SM, Cleeman JI, Merz CN, et al. Implications of recent clinical trials for the National Cholesterol Education Program Adult Treatment Panel III guidelines. Circulation 2004;110:227-39

3. McTaggart F, Buckett L, Davidson r, et al. Preclínical and clinical pharmacology of rosuvastatin, a new 3-hydroxy-3-methylglutaryl Coenzyme A reductase inhibitor. Am J Cardiol 2001;87:28B-32B

4. Konig J, Cui Y, Nies AT, et al. A novel human organic anion transporting polypeptide localized to the basolateral hepatocyte membrane. Am J Physiol Gastrointest Liver Physiol 2000;278:G156-64

5. Marzolini C, Paus E, Buclin T, et al. Polymorphisms in human MDR1 (P-glycoprotein): recent advances and clinical relevance. CLin Pharmacol Ther 2004;75:13-33

6. Staffa JA, Chang J, Green L. Cerivastatin and reports of fatal rhabdomyolysis. N Engl J Med 2002;346:539-40

7. Ucar M, Mjorndal T, Dahlqvist R. HMG reductase inhibitors and myotoxicity. Drug saf $2000 ; 22: 441-57$

8. Bliznakov EG. Lipid-lowering drugs (statins), cholesterol, and coenzyme Q10. The Baycol case - a modern Pandora's box. Biomed Pharmacother 2002;56:56-9
9. Rosenson RS. Current overview of statin-induced myopathy. Am J Med 2004;116:408-16

10. Jacobson TA. Comparative pharmacokinetic interaction profiles of pravastatin, simvastatin, and atorvastatin when coadministered with cytochrome P450 inhibitors. Am J Cardiol 2004;94:1140-6

11. Omar MA, Wilson JP. FDA adverse event reports on statin-associated rhabdomylolysis. Ann Pharmacother 2002;36:288-95

12. Lewin JJ III, Nappi JM, Taylor MH. Rhabdomyolysis with concurrent atorvastatin and diltiazem. Ann Pharmacother 2002;36:1546-9

13. Maltz HC, Balog DL, Cheigh JS. Rhabdomyolysis associated with concomitant use of atorvastatin and cyclosporine. Ann Pharmacother 1999;13:1176-9

14. Skaukat A, Benekli M, Vladutin GD, et al. Simvastatin-fluconazole causing rhabdomyolysis. Ann Pharmacoter 2003;37:1032-5

15. Fichtenbaum CJ, Gerber JG, Rosenkratz SL, et al. Pharmacokinetic interactions between protease inhibitors and statins in HIV seronegative volunteers: ACTG Study. AIDS 2002;16:569-77

16. Lilja JJ, Kivisto KT, Neuvonen PJ. Duration of effect of grapefruit on the pharmacokinetics of the CYP3A4 substrate simvastatin. CLin Pharmacol Ther 2000;68:384-90 
17. Bellosta S, Paoletti R, Corsini A. Safety of statins. Focus on clinical and pharmacokinetics and drug interactions. Circulation 2004;109:III50-III57

18. Prueksaritamont T, Tang C, Qiu Y, et al. Effects of fibrates on metabolism of statins in human hepatocytes. Drug Metab Dispos 2002;30:1280-7

19. Prueksaritamont $T$, Subramanian R, Fang $X$, et al. Glucuronidation of statins in animal and humans : a novel mechanism of statin lactonization. Drug Metab Dispos 2002;30:505-12

20. Kyrklund C, Backman JT, Neuvonen M, et al. Gemfibrozil increases plasma pravastatin concentrations and reduces pravastatin renal clearance. Clin Pharmacol Ther 2003;73:538-44

21. McFarlane SI, Muniyappa R, Francisco R, et al. Pleiotropic effects of statins: lipid reduction and beyond. Clin Endocrinol Metab 2002;87:1451-8

22. Kaneider NC, Egger P, Dunzendorfer S, et al. Rho-GTPase-dependent platelet-neutrophil interaction affected by HMG-CoA reductase inhibition with altered adenosine nucleotide release and function. Arterioscler Thromb Vasc Biol 2002;22:1029-35

23. Ishibashi T, Nagata K, Ohkawara H, et al. Inhibition of Rho/Rho-kinase signaling downregulates plasminogen activator inhibitor-1 synthesis in cultured human monocytes. Biochim Biophys Acta 2002;1590:123-30

24. Eto M, Kozai T, Cosentino F, et al. Statin prevents tissue factor expression in human endothelial cells: role of Rho/Rho-kinase and Akt pathways. Circulation 2002; 105:1756-9

25. Liao JK. Statin therapy for cardiac hypertrophy and heart failure. J Investig Med. 2004;52:248-53

26. Mehra MR, Raval NY. Metaanalysis of statins and survival in de novo cardiac transplantation. Transplant Proc 2004;36:1539-41

27. Leung BP, Sattar N, Crilly A, et al. A novel anti-inflammatory role for simvastatin in inflammatory arthritis. J Immunol 2003;170:1524-30

28. Sever PS, Dahlof B, Poulter NR, et al. Prevention of coronary and stroke events with atorvastatin in hypertensive patients who have average or lower-than-average cholesterol concentrations, in the AngloScandinavian Cardiac Outcomes Trial—Lipid Lowering Arm (ASCOT-LLA): a multicentre randomised controlled trial. Lancet 2003;361:1149-58

29. Colhoun HM, Betteridge DJ, Durrington $P N$, et al. Primary prevention of cardiovascular disease with atorvastatin in type 2 diabetes in the Collaborative Atorvastatin Diabetes Study (CARDS): multicentre randomised placebo-controlled trial. Lancet 2004;364:685-96

30. del Real G, Jimenez-Baranda S, Mira E, et al. Statins inhibit HIV-1 infection by down-regulating Rho activity. J Exp Med 2004;200:541-7

31. Schlienger RG, Meier CR. HMG-CoA reductase inhibitors in osteoporosis: do they reduce the risk of fracture? Drugs Aging 2003;20:321-36 